\title{
Towards an Automated Semantic Data-driven Decision Making Employing Human Brain
}

\section{Fensel, Anna}

Semantic Technology Institute (STI) Innsbruck, Department of Computer Science, University of Innsbruck, Austria

\begin{abstract}
Decision making is time-consuming and costly, as it requires direct intensive involvement of the human brain. The variety of expertise of highly qualified experts is very high, and the available experts are mostly not available on a short notice: they might be physically remotely located, and/or not being able to address all the problems they could address time-wise. Further, people tend to base more of their intellectual labour on rapidly increasing volumes of online data, content and computing resources, and the lack of corresponding scaling in availability of the human brain resources poses a bottleneck in the intellectual labour. We discuss enabling direct interoperability between the Internet and the human brain, developing "Internet of Brains", similar to "Internet of Things", where one can semantically model, interoperate and control real life objects. The Web, "Internet of Things" and "Internet of Brains" will be connected employing the same kind of semantic structures, and work in interoperation. Applying Brain Computer Interfaces (BCIs), psychology and behavioural science, we discuss the feasibility of a possible decion making infrastructure for semantic transfer of human thoughts, thinking processes, communication directly to the Internet.
\end{abstract}

Keywords: Semantic Technology; Decision Making; Brain Computer Inerface; Data Value Chain; Artificial Intelligence; Data Management. 


\section{Introduction}

Decision making is time-consuming and costly, as it requires direct intensive involvement of the human brain. The variety of expertise of highly qualified experts is very high, and the available experts are mostly not available on a short notice: they might be physically remotely located, and/or not being able to address all the problems they could address timewise. Generally, exchanging and managing the data on the Internet in a dynamic and efficient manner are among key challenges for the information systems requested nowadays by enterprises, institutions and citizens. People tend to base more of their intellectual labor on rapidly increasing volumes of online data, content and computing resources, and the lack of corresponding scaling in availability of the human brain resources poses a bottleneck in the intellectual labor. Finally, communication of the results of the intellectual labor requires further efforts, of putting the outcomes in a commonly processible representation form, such as spoken words or written texts.

To approach the optimal data management of the future, we discuss the possibility of enabling of direct interoperability between the Internet and the human brain, developing "Internet of Brains", similar to "Internet of Things". On the latter, one can semantically model, interoperate and control real life objects, and the applications of the semantic Internet of Things are numerous, see e.g. the areas of smart homes or transport. The Web, "Internet of Things" and "Internet of Brains" will be connected employing the same kind of semantic structures, and work in interoperation. Applying Brain Computer Interfaces (BCIs), psychology and behavioral science, an infrastructure for semantic transfer of human thoughts, thinking processes and communication directly to the Internet can be designed. This will facilitate the intellectual labor and its representation in human and machine readable forms, and address the aspects difficult to account so far, f.e. non-verbal communication.

Service-based enablers for discovery of interdependencies across human reasoning and senses and heterogeneous datasets for assisting humans in making decisions and changing their behavior and workflows can be created, as well as making these decisions and workflows more transparent and traceable. The latter can be performed taking into account the currently existing developments and standards in the related fields, particularly, semantic data licensing (Pellegrini et al., 2018), and smart contracts - as these are being exploited already broadly in practice (Underwood, 2016).

The high-level results of the envisioned solution will include:

- a framework for applying human thoughts and senses in decision making, through the semantic interfaces, and its concrete design and implementation,

- $\quad$ synthesizing concrete semantics from abstract thoughts and emotions, and 
- automation of an intellectual labor (in the way the robotics is replacing manual labor), with employment of these capacities.

And the corresponding technical objectives are as follows:

- Design and development of a semantic infrastructure capturing the domain of human reasoning and senses, as well as decision making and intellectual work processes that are based on them,

- Mapping the output of state of the art BCIs to the semantic infrastructure, producing a corresponding mappings library,

- With the framework for streaming human brain activity online, enabling easier modeling of the data in both design time and the run time of the digital workplace scenario - and eventually the organizations creating their own applications and workflows basing on these models,

- Speed up the velocity of the data flow in an information system that are currently bottlenecked by the slow speed of human decision making abilities, or are even performed with mistakes due to their imperfection (e.g. in scenarios connected with reporting),

- Making the decision processes transparent, traceable, and easier to optimize (e.g. it can be easily established which nodes are causing delays),

- Integrate new techniques facilitating easier data reuse, such as semantic information on how the data and content can be licensed (licenses library and tools can be applied out of our development in DALICC project $^{1}$ ),

- Visualization of the data, decisions in a form that is actionable to humans in a digital workplace scenario.

\section{State of the Art and Progress Beyond it}

The proposed solution will aim to advance the state of the art in the following areas: (1) semantic modelling, knowledge representation, (2) data-empowered reasoning and decision making, (3) sensor technology. Further, we overview of the state of the art in these fields and how the aimed results are expected to advance the state of the art.

\footnotetext{
${ }^{1}$ DAta LIcenses Clearing Center: https://dalicc.net
} 


\subsection{Semantic Technology as a Communication Means on the Internet}

"The Semantic Web is not a separate Web but an extension of the current one, in which information is given well-defined meaning, better enabling computers and people to work in cooperation." - this statement of Tim Berners-Lee has gained even more relevance since the start of this century (Berners-Lee et al., 2001). The vision of the Semantic Web seminal paper came closer, starting with the appearance of the basic semantic languages such as RDF, RDFS, OWL, semantic web service languages. Early from the appearance of the Semantic Web, the challenge to use semantics to facilitate human communication is being addressed, with the "semantic desktop" initiative and NEPOMUK project (Decker and Frank, 2004) being among the first ones. Now there exist IT multi-stakeholder ecosystems and infrastructures to interoperate across different marketing data and content resources using Linked Data (Bizer et al., 2009) and semantic technologies (Domingue et al., 2011), enhancing interoperability of distributed resources for allowing meaningful searches and efficient information dissemination for humans alike as machines. Research and developments on combining human and computing resources are abundant - see e.g. developments such as "social machines" (Hendler and Mulvehill, 2016), and use cases of that kind in the infrastructures such as Wikipedia (Smart et al., 2014), however, none of them yet comprise processing of the direct input from the human brain.

In this development, skipping of the step involving natural language processing technology and communicating the outcomes of human thinking to machines in a semantic form will become possible. This will be used in the first place by the people with limited abilities, but also by people who are able to represent their thinking in an intermediate representation format (spoken words, written texts, etc.) - for the reasons of efficiency and scalability.

In research and commercial developments, methods and tools to extract semantics from intermediary communication means have been developed, for example, extracting emotions and sentiments from the Web (Baldoni et al., 2012), as well as from the natural language texts (Mathieu, 2005). Such methods work already work in practice with a relatively large success, but inherently presume the availability of intermediary knowledge representation sources. On the contrary, here, extraction of emotions and sentiments from the human brain would take place directly. Some of the modeling and representation of the sentiments and emotions from the state-of-the-art research can be taken into account when modelling the framework, also including dedicated efforts to build the relevant ontologies (López et al., 2008; Borth et al., 2013).

\subsection{Reasoning and Decision Making in the "Data Tsumami" Conditions}

As it is known, in the human reasoning and decision making, there are normally "soft" and "hard" factors involved. For example, if someone is hiring an employee to work with, usually both sides are important: whether a potential employee has an adequate 
qualification and experience ("hard" factors), and whether he or she would fit well in the team ("soft" factors). Frameworks and models are currently starting to appear in the literature in application to various tasks and domains e.g. forecasting (Bańbura \& Rünstler, 2011), as well as the approaches towards explaining human decision making (Rosenfeld and Kraus, 2018). Such works approach the possibilities to formalize the decision making and reasoning process semantically.

In the world overflown by a "data tsunami", humans are standing at the edge of their decision making and behavior change capacities, and the need to overcome these is unavoidable. The reasons here are as follows:

- Drastic increase in the amounts of the data and information that can be potentially relevant for making right decisions, de facto, the current "hard" reasoning we perform now is mostly always "incorrect and incomplete" - and methods and tools to address such reasoning (Fensel et al., 2008) have been developed, particularly, in EU LarKC project $^{2}$,

- Limitations and restrictions of human mind in taking decisions (such as due its limited immediate storage capacity, irrationality caused by bias (Boutang and De Lara, 2015)),

- Increase in the dynamicity: often, the situations change on the fly, and the used data, workflow models may need to be replaced - as well as the behavior changed, this again, poses a challenge in choice of goals, methods, and implementation of tasks to a human brain,

- Effective decisions and human behavior changes are essential parts of success, in particular economic success; even more dramatic: in some areas such as climate change or energy efficiency, the change of human behavior may mean the difference between "to be" and "not to be" for the human kind.

Given the ability to process and analyze large amounts of data, the machines already arguably outperform humans when it comes to the intellectual labor, where only "hard" factors are involved. However, many decisions carried out solely on the "hard" facts remain unviable in the real world, as they may go in contrary with the human senses, emotions, feelings, intuition, and eventually safety of the humans and acceptance with them. Applying on emotions (fear, curiosity, enjoyment and many others), human brains are able to rather successfully filter out the "right" contexts and defining the new ones (Kahneman, 2011), i.e. possessing the "soft factor" capacity which machines do not possess.

\footnotetext{
${ }^{2}$ Large Knowledge Collider: http://larkc.sti2.at (archived web site)
} 
Online communication, on the other hand, is not trivial, as it still hides most, or a large part of the semantics, e.g. transferred over non-verbal communication in face-to-face communication. Leaving alone the fact that a human, in order to communicate, needs to create a representation of the thought or an emotion, e.g. spoken words, images, text, which is of course not $100 \%$ identical to the original thought or an emotion. Here, we will pursue elimination of the typical intermediate representation layer for a human brain activity, and will create a precise machine and human readable semantic layer for it instead, and map the signals coming out of the bio-sensing equipment directly into this layer. In communication infrastructures, heterogeneous communities of stakeholders need to be addressed, and semantics is a very suitable instrument for this, as the essence of ontologies inseparably reflect the communities using them (Mika, 2005; Zhdanova, 2008).

An additional challenge here is that humans also have a tendency to conceal the outcomes of their thinking, or even communicate the facts that do not correspond to them, if they feel like they would be getting an advantage, in particular, a match to the desired limited resource on the market (Roth et al., 2015), or a better perception by the society. Again, semantics has a potential to resolve this challenge, and test/simulate the realities which would take place under the conditions of humans expressing their actual thoughts and feelings.

\subsection{Hardware and Sensors Availability}

A better understanding of the human brain stands high on the priority of the European Commission, e.g. The Human Brain Project ${ }^{3}$ is ongoing as a H2020 FET Flagship Project which strives to accelerate the fields of neuroscience, computing and brain-related medicine, since 2013, with the duration of 10 years. On the side of the technical development, also the BCIs have been investigated, and the forecasts and scenarios have been roadmapped, confirming the expected broad spread and varying spectrum of the application scenarios where BCIs will be used (Brunner et al., 2015).

Now it is the right time to base the project on this technology, due to the following technical reasons:

1) Now the BCI technology is becoming mature and available. Companies selling advanced consumer-oriented products in the sub-1000 dollar range include Emotiv (http://www.emotiv.com) and Neurosky (http://www.neurosky.com). There are also very inexpensive open source biosensing solutions, such as OpenBCI (http://openbci.com). Generally, currently tools for make technical connections to the brain cost as little as starting from 30 dollars.

\footnotetext{
${ }^{3}$ Human Brain Project: https://www.humanbrainproject.eu/en/
} 
2) The user acceptance level of the technology is also becoming sufficient, to have an expectation that a system developed on a base of a BCI will be used and usable.

Now BCI are already actively used beyond the typical for them medical scenarios, and e.g. are employed in games - however, also there a systematic approach of the related data management is missing (Gurkok et al., 2017). Thus, a systematic approach for integrating human thinking and reasoning activity on the Internet needs to be designed and developed practically.

\section{Conclusions}

We have described initial principles and prerequisites of the direct integration of the data stemming from the human brain into decision making processes of the future. The main measurable success criterion of this work can be characterized as a transition step from Big Data to Smart Data. It typically involves enablement of more efficient adding value participation, i.e. increasing efficiency and/or provisioning and take up of new types of interactivity which bring benefits to the involved stakeholders (for example, faster decision making, less effort to transform the brain activity into the intermediary communication formats such as spoken words or written text). Further, the societal externalities of Big Data use (Cuquet and Fensel, 2018) will be accounted for, even when humans are unaware of them. The approach is to be realized in the chosen application domains going beyond the current state of the art of ontology-based service interfacing, integration and bio- and crowd- sensing. The results are to be evaluated within real scenarios, with real life data and services, as well as with real end users. The evaluation outcomes are to confirm the technical feasibility of ontology-based intervention networked services enablement, as well as its added value from the originated new usage scenarios, and its acceptance by the end users.

\section{Acknowledgements}

This work has been partially funded by project DALICC, supported by the Austrian Research Promotion Agency (FFG) within the program "Future ICT".

\section{References}

Baldoni, M., Baroglio, C., Patti, V., \& Rena, P. (2012). From tags to emotions: Ontologydriven sentiment analysis in the social semantic web. Intelligenza Artificiale, 6(1), 4154. 
Bańbura, M., \& Rünstler, G. (2011). A look into the factor model black box: publication lags and the role of hard and soft data in forecasting GDP. International Journal of Forecasting, 27(2), 333-346.

Berners-Lee, T., Hendler, J., \& Lassila, O. (2001). The semantic web. Scientific American, 284(5), 34-43.

Bizer, C., Heath, T., \& Berners-Lee, T. (2009). Linked data-the story so far. Semantic Services, Interoperability and Web Applications: Emerging Concepts, 205-227.

Borth, D., Chen, T., Ji, R., \& Chang, S. F. (2013, October). Sentibank: large-scale ontology and classifiers for detecting sentiment and emotions in visual content. In Proceedings of the 21st ACM international conference on Multimedia, 459-460. ACM.

Boutang, J., \& De Lara, M. (2015). The Biased Mind: How Evolution Shaped Our Psychology Including Anecdotes and Tips for Making Sound Decisions. Springer.

Brunner, C., Birbaumer, N., Blankertz, B., Guger, C., Kübler, A., Mattia, D., del R. Millán, J., Miralles, F., Nijholt, A., Opisso, E., Ramsey, N., Salomon, P., \& Müller-Putz, G.R. (2015) BNCI Horizon 2020: towards a roadmap for the BCI community. BCI Journal. URL: http://bnci-horizon-2020.eu/roadmap

Cuquet, M., \& Fensel, A. (2018). The societal impact of big data: A research roadmap for Europe. Technology in Society, Elsevier.

Decker, S., \& Frank, M. (2004). The social semantic desktop. Digital Enterprise Research Institute, DERI Technical Report May, 2, 7.

Domingue, J., Fensel, D., \& Hendler, J. A. (Eds.). (2011). Handbook of semantic web technologies (Vol. 1). Springer Science \& Business Media.

Fensel, D., Van Harmelen, F., Andersson, B., Brennan, P., Cunningham, H., Della Valle, E., Fischer, F., Huang, Z., Kiryakov, A., Kyung-il Lee, T., Schooler, L., Tresp, V., Wesner, S., Witbrock, M., \& Zhong, N. (2008). Towards LarKC: a platform for webscale reasoning. In IEEE International Conference on Semantic Computing, 524-529, IEEE.

Gurkok, H., Nijholt, A., \& Poel, M. (2017). Brain-Computer Interface Games: Towards a Framework. Handbook of Digital Games and Entertainment Technologies, 133-150.

Hendler, J., \& Mulvehill, A. M. (2016). Social machines: the coming collision of artificial intelligence, social networking, and humanity. Apress.

Kahneman, D. (2011). Thinking, fast and slow. Macmillan.

López, J. M., Gil, R., García, R., Cearreta, I., \& Garay, N. (2008, September). Towards an ontology for describing emotions. In World Summit on Knowledge Society, 96-104, Springer, Berlin, Heidelberg.

Mathieu, Y. Y. (2005, October). Annotation of emotions and feelings in texts. In International Conference on Affective Computing and Intelligent Interaction, 350-357, Springer, Berlin, Heidelberg.

Mika, P. (2005). Ontologies are us: a unified model of social networks and semantics. In: Proceedings of the 4th International Semantic Web Conference, LNCS 3729, 522-536, Springer.

Pellegrini, T., Schönhofer, A., Kirrane, S., Steyskal, S., Fensel, A., Panasiuk, O., MirelesChavez, V., Thurner, T., Dörfler, M., \& Polleres, A. (2018). A Genealogy and 
Classification of Rights Expression Languages - Preliminary Results. In: Trend and Communities of Legal Informatics - Proceedings of the 21st International Legal Informatics Symposion, IRIS 2018, 243-250, Salzburg, Austria.

Rosenfeld, A., Kraus, S. (2018). Predicting Human Decision-Making: From Prediction to Action. Morgan and Claypool.

Roth, A. E. (2015). Who Gets What—and Why: The New Economics of Matchmaking and Market Design. Houghton Mifflin Harcourt.

Smart, P., Simperl, E., \& Shadbolt, N. (2014). A taxonomic framework for social machines. In Social Collective Intelligence, 51-85, Springer, Cham.

Underwood, S. (2016). Blockchain beyond bitcoin. Communications of the ACM, 59(11), 15-17.

Zhdanova, A.V. (2008). Community-driven Ontology Construction in Social Networking Portals. International Journal on Web Intelligence and Agent Systems, 6(1), 93-121, IOS Press. 